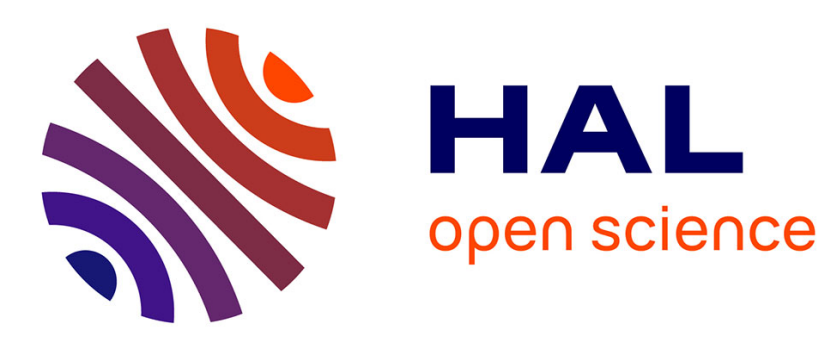

\title{
FES-controlled Co-contraction Strategies for Pathological Tremor Compensation
}

\author{
Antonio Bo, Philippe Poignet, Dingguo Zhang, Wei Tech Ang
}

\section{To cite this version:}

Antonio Bo, Philippe Poignet, Dingguo Zhang, Wei Tech Ang. FES-controlled Co-contraction Strategies for Pathological Tremor Compensation. IROS: Intelligent RObots and Systems, Oct 2009, St. Louis, MO, United States. pp.1633-1638, 10.1109/IROS.2009.5354397 . lirmm-00412947

\section{HAL Id: lirmm-00412947 https://hal-lirmm.ccsd.cnrs.fr/lirmm-00412947}

Submitted on 2 Sep 2009

HAL is a multi-disciplinary open access archive for the deposit and dissemination of scientific research documents, whether they are published or not. The documents may come from teaching and research institutions in France or abroad, or from public or private research centers.
L'archive ouverte pluridisciplinaire HAL, est destinée au dépôt et à la diffusion de documents scientifiques de niveau recherche, publiés ou non, émanant des établissements d'enseignement et de recherche français ou étrangers, des laboratoires publics ou privés. 


\title{
FES-controlled Co-contraction Strategies for Pathological Tremor Compensation
}

\author{
Antônio Padilha Lanari Bó, Philippe Poignet, Dingguo Zhang, Wei Tech Ang
}

\begin{abstract}
In this paper, a strategy for pathological tremor compensation based on co-contraction of antagonist muscles induced by Functional Electrical Stimulation (FES) is presented. Although one of the simplest alternatives to apply FES for reducing the effects of tremor, the contribution of different cocontraction levels for joint motion and impedance must be accurately estimated, specially since tremor itself is highly time-varying. In this work, a detailed musculoskeletal model of the human wrist actuated by flexor and extensor muscles is used for this purpose. The model takes into account different properties that affect muscle dynamics, such as proprioceptive feedback and combined natural and artificial activation. The model, analysis of stiffness modulation due to FES-controlled co-contraction and simulation results are presented in the paper.
\end{abstract}

\section{INTRODUCTION}

Tremor may be defined as an involuntary, approximately rhythmic and roughly sinusoidal movement [1]. Although pathological tremor itself is not lifethreatening, it may decrease considerably the person's ability to perform simple daily tasks, especially since its incidence is higher on the upper limb. It is the most common movement disorder found in human pathology.

The pathogeneses of most types of tremor are still unclear and an absolutely effective treatment is not yet available. Pharmacological and surgical therapies exist for the two most prevalent types of tremor, essential tremor and the tremor associated with Parkinson's Disease, but they still present limitations. In this context, one alternative is the use of assistive devices to reduce the effects of the abnormal motion. Among the new technologies, upper limb robotic exoskeletons [2] and the use of FES [3] have been proposed.

In order to build such a functional assistive device, some design challenges must be overcome. The robotic system must, for instance, adapt itself regularly, since tremor often presents highly time-varying dynamics. Also, it must be able to filter voluntary motion from pathological motion, in order to avoid compensating the action the person is willing to execute. Finally, the system must be built in such a way that the person may still be able to conduct normal activities comfortably, i.e., it must present low weight and appealing cosmetic

Antônio P. L. Bó (e-mail: bo@lirmm.fr) and Philippe Poignet are with the LIRMM UMR 5506 CNRS UM2, France. Antônio P. L. Bó is also with Neuromedics, France. Dingguo Zhang is with Shanghai Jiao Tong University, China. Wei Tech Ang is with Nanyang Technological University, Singapore. features and cause little fatigue or pain. Considering these requisites, this work was conducted with the goal of evaluating the use of surface Functional Electrical Stimulation (FES) for tremor compensation.

One of the simplest strategies to reduce the effects of pathological tremor is to artificially induce cocontraction of antagonist muscles that act on the trembling joint. Co-contraction of antagonist muscles is one of the strategies that we employ in tasks that require more precision and stability, since the joint stiffness is increased. However, to apply this strategy in real applications, one must quantify both the muscles contribution to joint impedance and the co-activation levels for which the net torque produced is zero, such that voluntary motion is not disturbed.

In this work, a detailed mathematical model of the system is employed, due to the system complexities and nonlinearities. We use a musculoskeletal model of the human wrist joint actuated by different flexor and extensor muscles, which presents as inputs both natural, composed by pathological and voluntary components, and artificial muscle excitations. In addition to the computation of the referred properties, the model may also be used in dynamic simulations of FES-controlled co-contraction tremor compensation, before real experiments are conducted with patients. Furthermore, this model may be useful in the design of model-based closedloop systems for the suppression of tremor and also help in the studies of different compensation strategies.

Musculoskeletal modeling has been an active research field for many years. Such models have been applied in many research areas, including theoretical tremor studies [4]. However, most of the related literature does not concern electrically stimulated muscles. Indeed, the need for the establishment of reliable models for FEScontrolled muscles has been pointed out in [5]. One of the current approaches, described in [6], is the Hill-based muscle model [7].

Another contribution of the paper lies in the developed model. Although based in a similar approach, the model tries to address some limitations related to the model proposed in [6], such that muscle active viscoelasticity is more accurately represented. For instance, proprioceptive feedback, which is known to affect muscle contribution to joint impedance, is considered. Also, some other features are needed to take into account muscle combined activation due to both natural activation and FES.

This paper is organized as follows. Section II presents 


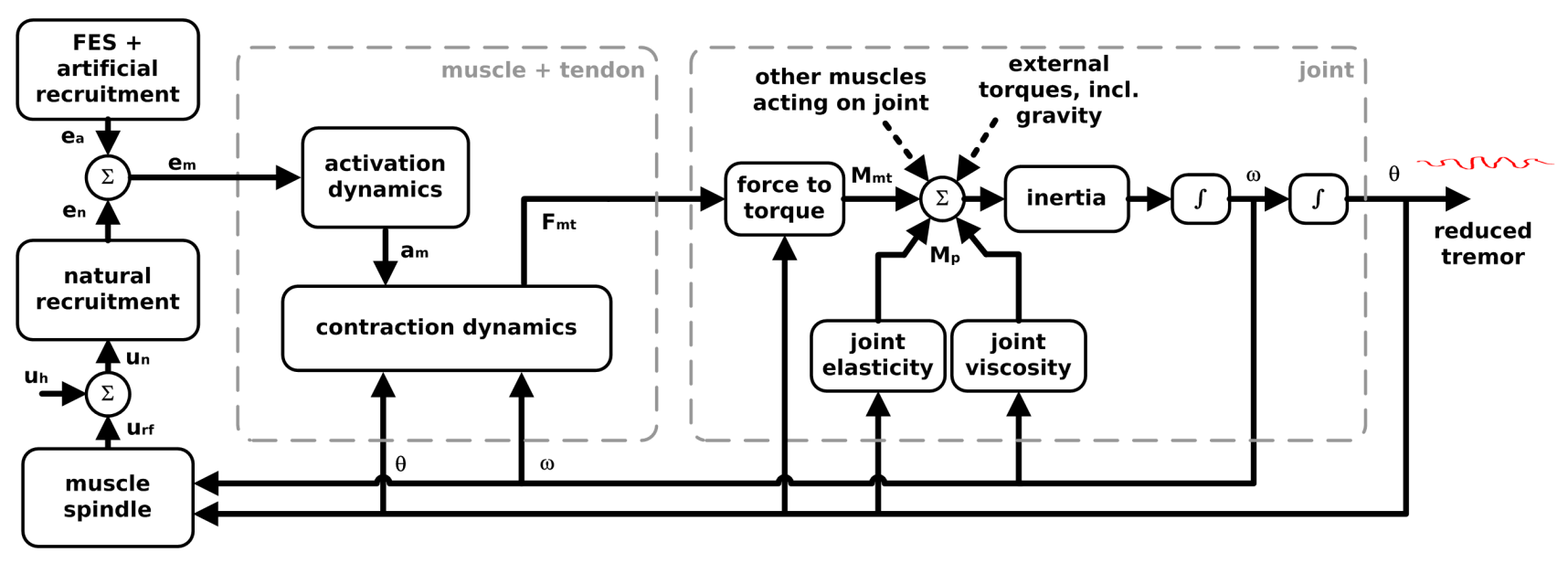

Fig. 1. The structure of the musculoskeletal model presented in this paper. Only one muscle is shown.

the wrist model. Joint dynamics, musculotendon contraction, activation dynamics and proprioceptive feedback are described in different subsections. In the following section, experimental data and simulation results that illustrate tremor reduction via FES-controlled cocontraction are shown. Finally, an analysis of the contribution of multiple muscles to the wrist stiffness is performed. Final remarks are presented on the last section.

\section{Model Structure}

To describe the behavior of a human joint due to the concerned muscles and their respective neural inputs (or electrical stimulation) is a complicated task. The force generated by the muscle is a nonlinear function of the neural input, but also depends on the muscle length, velocity, its level of fatigue and more. Figure 1 illustrates the model structure adopted in this work.

The model has parameters that are specific to a certain musculotendon actuator and others that are common to different muscles. In that context, a dimensionless musculotendon model [7], is scaled by the specific parameters [8], [9]. Here, the variables presented are normalized, with the normalization carried out according to [7].

\section{A. Wrist joint}

Due to the high incidence of tremor at the wrist level and the functional importance of that joint, this type of tremor is a major source of discomfort for tremor patients. Furthermore, the wrist is a feasible and simple target for tremor compensation systems based on orthoses or FES, as opposed to the fingers, and hence it has been chosen to be used in this simulation study.

The wrist model is simply given by

$$
J \ddot{\theta}=M_{m t f}-M_{m t e}-M_{p}+M_{g}+M_{e},
$$

where $\theta$ is the wrist angle, $J$ is the inertia for flexion/extension, $M_{p}$ is the sum of internal passive moments on the wrist which are independent from the muscles, $M_{g}$ is the moment due to gravity, and $M_{m t f}$ and $M_{m t e}$ are the moments resulting from flexor and extensor muscles actions, respectively. $M_{e}$, the moment due to external forces, was used to allow evaluation of different compensation strategies, like attaching an inertial load to the hand, and the overall stability.

To compute the torques due to the musculotendon forces acting on the wrist, instead of using a geometrical model to compute the muscle arms, we employed the polynomial functions described in [9], which were based on data previously published by [8]. From the same source, we obtained the relations to compute musculotendon lengths as a function of the wrist angle.

To compute $M_{p}$, the model proposed in [7] was used. It is an attempt to model effects caused by different tissues and also bony constraints. It is given by

$$
M_{p}=k_{p} \theta+b_{p} \dot{\theta}+k_{p g}\left(e^{k_{p e} \theta}-1\right),
$$

where $k_{p}, b_{p}, k_{p g}$, and $k_{p e}$ are constant parameters.

\section{B. Musculotendon contraction}

Musculotendon contraction dynamics is often described in terms of Huxley-based models, which take into account the physiological description of the contraction, and Hill-based models, which offers a more systemic point of view. In our case, a Hill-based model is chosen, since it represents a good compromise between complexity and tractability. For some applications, even simpler models are considered [10], [6], but in the present model further simplifications are not desired, since they could not represent the full range of expected inputs and neglect the nonlinear dynamics of muscle action.

In a Hill-based model, the muscle is composed of an active Contractile Element (CE) and two passive components: a parallel element (PE), which mainly represents muscle passive elasticity, and a serial element (SE), related in this work to tendon elasticity. One possible arrangement of these elements is shown on Fig. 2.

The implementation of the forward dynamics of the muscle model was based on [7], where the computations are made with normalized units. The force supplied by 
the $\mathrm{CE}$, which is normalized by the muscle maximum isometric force, is given by:

$$
F_{c e}=a_{m} f_{l}\left(l_{m}, a_{m}\right) f_{v}\left(v_{m}, a_{m}\right),
$$

where $a_{m}$ is the active state of the muscle, $l_{m}$ is the muscle length, $v_{m}$ its velocity, and $f_{l}$ and $f_{v}$ represent respectively the force-length and the force-velocity, described below. Accurate $f_{l}$ and $f_{v}$ functions are particularly important if one attempts to represent the muscle contribution to joint stiffness. $f_{l}$ curve is given by

$$
f_{l}=e^{-\left(\frac{l_{m}-l_{\max }}{l_{s h}}\right)^{2}},
$$

where

$$
l_{\text {max }}=l_{o o}+l_{\text {shft }}\left(1-a_{m}\right),
$$

and $l_{s h}, l_{o o}$, and $l_{s h f t}$ are constant parameters.

The force-velocity contribution to muscle force may be computed according to:

$$
f_{v}= \begin{cases}0 & , v_{m}<-v_{\max } \\ \frac{v_{s h}\left(v_{\max }+v_{m}\right)}{v_{s h} v_{\max }-v_{m}} & ,-v_{\max } \leq v_{m} \leq 0 \\ \frac{v_{s h} v_{\operatorname{shl} l v_{\max }+v_{\operatorname{ml}} v_{m}}}{v_{s h} v_{s h l} v_{\max }+v_{m}} & , v_{m}>0,\end{cases}
$$

where

$$
v_{\text {max }}=v_{v m}\left[v_{\text {rat }}+a_{m}\left(1-v_{\text {rat }}\right)\right],
$$

and $v_{s h}, v_{s h l}, v_{m l}, v_{v m}$, and $v_{r a t}$ are constant parameters.

In a Hill-based muscle model, normally not only the active component presents nonlinear behavior, but also the passive components. In this work, both $\mathrm{PE}$ and SE are described by elastic nonlinear functions, but do not present viscous behavior. The force generated by the PE is given by

$$
F_{p e}=\frac{1}{e^{p e_{s h}}-1}\left[e^{-\frac{p e_{s h}}{p e_{x m}}\left(l_{m}-1\right)}-1\right],
$$

where $p e_{s h}$ and $p e_{x m}$ are constant parameters.

Since CE and PE are placed in series with SE in this model, the force produced by $\mathrm{SE}, F_{s e}$, is equal to $F_{c e}+$ $F_{p e}$. It is a function of tendon strain, $\epsilon_{s e}$ :

$$
F_{s e}= \begin{cases}\frac{1}{e^{s e_{s h}-1}}\left(e^{\frac{s e_{s h}}{\epsilon_{s e}} \epsilon_{s e}}-1\right) & , \epsilon_{s e} \leq \epsilon_{s e}^{0} \\ \sigma_{s e}^{0}+k_{s e}\left(\epsilon_{s e}-\epsilon_{s e}^{0}\right) & , \epsilon_{s e} \geq \epsilon_{s e}^{0},\end{cases}
$$

where $\epsilon_{s e}^{0}$ and $k_{s e}$ are muscle dependent parameters and $s e_{s h}$ is a constant parameter.

In some musculoskeletal models, like [6], the tendon is not included (i.e., SE is considered infinitely stiff). Forward simulation of contraction dynamics is simplified, since the force may be directly computed from the provided $l_{m}$ and $v_{m}$. In our case, at each iteration the muscle velocity is estimated with the Newton-Raphson method, and then used to estimate muscle and tendon lengths. The effort to consider the compliant tendon was particularly important because of the long tendons presented by the forearm muscles [8].

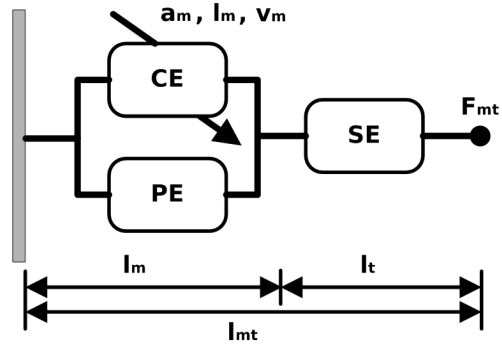

Fig. 2. Hill-based musculotendon unit.

\section{Muscle activation}

In general, musculoskeletal models used for FES control do not include the combined stimulation from natural and artificial stimuli. This is so because most of these models are applied to motor restoring for individuals with spinal cord injury (SCI), and hence the effects of reflex loops on muscle contraction dynamics may be partially included in the muscle model or simply neglected. In our work, however, the main use of the musculoskeletal model is in the compensation of pathological tremor, i.e., for patients that possess full control of muscle contraction. Also, since a good representation of muscle contribution to joint stiffness is desired, an accurate model of proprioceptive feedback is equally needed.

The natural stimulation represents the output from the $\alpha$-motoneurons (MNs), which is a function of the total neural input to the MN, $u_{n}$. $u_{n}$ is given by the sum of the neural drive from higher levels of the central nervous system (CNS), $u_{h}$, and the neural drive from the reflex loops, $u_{r f}$. To represent the muscle excitation level due to natural stimuli, $e_{n}$, as a function of $u_{n}$, we apply, as in [7], a first-order transfer function, which represents both the $\alpha$-MN dynamics and the neural transmission:

$$
\dot{e}_{n}=\frac{u_{n}-e_{n}}{T_{e n}},
$$

where $T_{e n}$ is the time-constant.

Concerning the muscle excitation due to the surface FES, $e_{a}$, it is common to compute it from the stimulation parameters, and not the signal itself, which is normally composed by a train of biphasic pulses. Another common approach is to model separately the dynamics of a single motor unit (temporal recruitment) and the recruitment level of the several motor units that compose a particular muscle (spatial recruitment). Once $e_{a s}$, that represents spatial recruitment, and $e_{a t}$, that represents temporal recruitment, have been computed, the total FES input, $e_{a}$, may be given by the product of the two factors.

To represent the recruited motor units as a function of stimulation amplitude, $i$, or pulse width, $p$, some works employ sigmoid-shape static functions [6]. In this work, a recruitment function that takes into account varying $i$ and $p$ [11] is used, since both $i$ and $p$ may control the number of recruited motor units.

Concerning the excitation dynamics of a single motor 
unit, it is often computed directly from the stimulation frequency, $f$. This is the case specially for systems where $f$ is kept constant or varies over tetanic stimulation frequencies, like [6]. In our case, however, we would like to be able to apply a broader range of stimulation frequencies. Hence, the alternative is to use the stimulation signal itself to represent temporal recruitment. In this work, a first-order transfer function was used:

$$
\dot{e}_{a t}=\frac{k_{e a} u_{a}-e_{a t}}{T_{e a}},
$$

where $T_{e a}$ and $k_{e a}$ are, respectively, the time-constant and the gain, for which different values are assigned when $u_{a}>e_{a t}$ and $u_{a}<e_{a t} . u_{a}$ is the stimulation signal, with pulse amplitudes scaled to unity.

Since both inputs have been scaled down to coherent values, the total excitation level of the muscle, $e_{m}$, may be given by the sum of both natural and artificial inputs. This approximation neglects some aspects of FES, especially the inverse recruitment order, but it has been assumed for the moment.

Finally, the active state of the muscle may be computed from the muscle excitation level. The activation dynamics represents the physiological events that occur from the moment the stimulus arrives until the muscle cell contracts. This process, as in [7], may be also described by first-order dynamics, with slower and different time-constants for activation and de-activation.

\section{Muscle spindle}

In order to improve the accuracy of musculoskeletal models, some works have included the effects of lowlevel spinal reflexes on muscle contraction. Nevertheless, the effects of proprioceptive feedbacks on musculotendon dynamics are a controversial subject [7]. For instance, the roles of the golgi tendon organ (GTO) and the Renshaw cell (RC) are still a matter of debate, and hence are not considered. However, since the effects of muscle spindles are seen as important for motor control, especially in terms of stiffness and disturbance rejection, we have chosen to include them in the model.

Muscle spindles are often seen as sensors that provide information about muscle length and muscle velocity. More specifically, they are sensory receptors located in sheathed muscle fibers (called intrafusal muscle fibers) that lie in parallel with the extrafusal fibers. Those special muscle fibers are innervated by $\gamma-\mathrm{MNs}$, which modulate the muscle spindle sensibility.

In our work, a model similar to the one proposed in [7] is used. The intrafusal contraction is computed as for the extrafusal fibers, with the main difference that the SE does not represent the tendon, but the non-contractile element where tension is measured. In this model, $\alpha$ $\gamma$ co-activation is assumed, which is an hypothesis to describe the muscle spindle sensibility modulation in regular motor tasks. $u_{r f}$, the output from the muscle

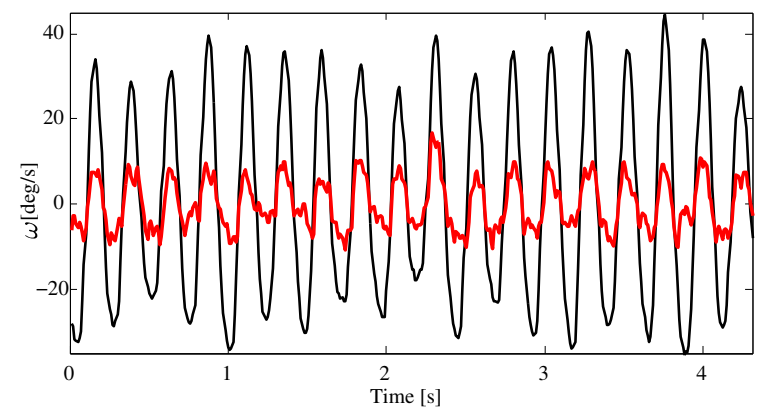

Fig. 3. FES-induced tremor of the wrist via Extensor Digitorum Communis (EDC) stimulation before (black) and after (red) Extensor Carpi Radialis (ECR) and Flexor Carpi Radialis (FCR) co-contraction by means of surface stimulation. The experiment was conducted in a healthy subject with a commercial stimulator, the Cefar Physio 4, from CefarCompex. The wrist motion was measured with an angular rate sensor placed on the hand.

spindle, is given by

$$
u_{r f}=k_{r f s} \epsilon_{i f}+k_{r f d} \dot{\epsilon}_{i f},
$$

where $\epsilon_{i f}$ is the strain at the sensory region, and $k_{r f s}$ and $k_{r f d}$ are constant gains. Also, a time-delay is included to represent the neural transmission.

\section{FES-CONTROLLED CO-CONTRACTION FOR TREMOR COMPENSATION}

Co-contraction of antagonist muscles is one of the strategies employed by the CNS in tasks that require more precision and stability, since the joint stiffness is increased. In fact, artificially inducing co-contraction is one of the simplest strategies to reduce the effects of pathological tremor, as illustrated in Fig. 3.

However, to be useful in real applications, the net torque produced by these different co-contraction levels must be zero, so that voluntary motions are minimally affected. Furthermore, impedance modulation must be continuously corrected, since tremor is a time-varying motion. Once those relations are estimated, impedance control strategies, like the one described in [2] for exoskeleton-based tremor suppression, may be applied. For a FES-based compensation system, however, this function takes a more complicated form when compared to the exoskeleton solution. Some works have simply considered that muscle active stiffness is proportional to muscle force [12]. Here, the detailed nonlinear musculoskeletal model developed is used to describe this effect.

In this section, first simulations are described in which the model described in Section II is used to illustrate tremor reduction by co-contraction of antagonist muscles. Next, an analysis of joint stiffness control by FEScontrolled co-contraction is presented.

\section{A. Simulating tremor reduction}

Based on the model described in Section II, simulations were performed to illustrate tremor reduction via cocontraction of antagonist muscles induced by FES. Fur- 


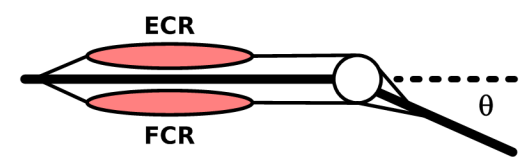

Fig. 4. Wrist model used in the simulations, with ECR and FCR as the pair of antagonist muscles.

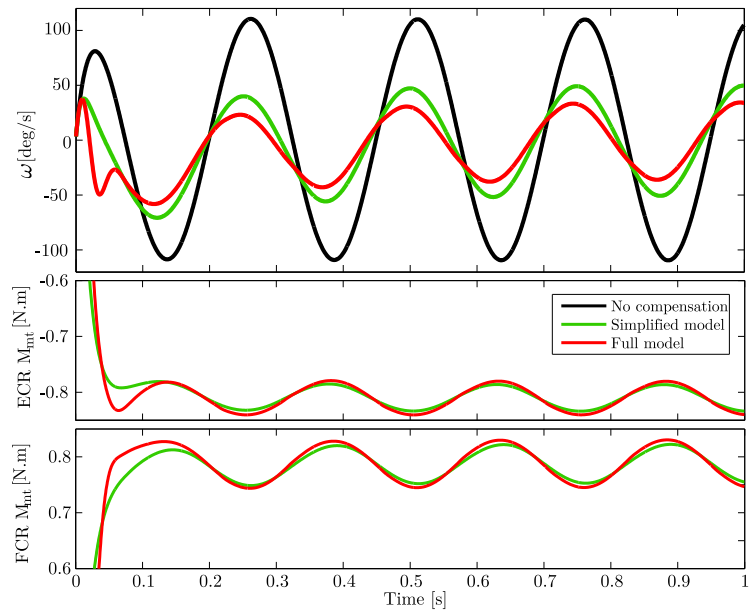

Fig. 5. Simulated trembling wrist: without any muscle action (black) and with muscle forces computed with the full model described in Section II (red) and with a simplified model (green).

thermore, tremor reduction predicted by different models are briefly compared.

In this study, the chosen muscles chosen are the ECR, composed by Longus and Brevis portions, and the FCR, two of the most important extensors and flexors of the wrist, respectively. In addition, we have observed that those two muscles generally present a good response to surface stimulation, while the electrode placement procedure is simple if compared to other muscles of the forearm. The same muscles were stimulated in the experiment illustrated in Fig. 3. Figure 4 represent the model used in the simulations.

Tremor was represented by a sinusoidal external moment acting on the joint. Both the tremor amplitude and the co-activation level were kept constant. In a real application, the co-activation level must be controlled not only by tremor time-varying features, but also by the patient-specific discomfort level caused by FES.

The results are illustrated in Fig. 5. Wrist motion with higher amplitude occurs when there is no muscle action. For muscle models that do not consider muscle viscoelasticity, the same output would be observed. The figure also shows the response using two different muscle models: the full model described in Section II and a simplified model (without a compliant tendon and any proprioceptive feedback). The same FES stimulation parameters were applied to both models. As expected, the simplified model underestimates muscle contribution to joint impedance. Phase differences are due to the different dynamics included in each simulation.

\section{B. Wrist stiffness control via FES-controlled co- contraction}

Since experimental and simulation data support tremor compensation via co-activation of antagonist muscles, now we present an analysis of FES to the control the muscles contributions to wrist stiffness.

Firstly, based on the nonlinear model described in Section II, the equilibrium wrist angles due to different co-activation levels (Fig. 6) were numerically computed. Each co-activation level, represented by $\mathbf{c}$, is defined by the pair of muscle excitations due to FES stimulation, $e_{a f}$ and $e_{a e}$ for flexor and extensor muscles, respectively. Since $e_{a f} \in[0,1]$ and $e_{a e} \in[0,1]$, the set of all cocontraction levels was discretized for computation.

A similar strategy was chosen in [13], where steadystate angles are computed and used for motion control. In our case, however, estimating all equilibrium points is also important to determine which stimulation combinations do not produce flexion or extension of the wrist. To express this notion mathematically, the following procedure was used. The intersection between the set of all equilibrium points and the plane in which the final equilibrium angle is zero, the red line featured on Fig. 6 , produces the set $\mathbf{C}_{0} \subset \Re^{2}$ of co-activation levels $\mathbf{c}_{\mathbf{0}}$. Then, a normalized function $f_{0}\left(u_{c 0}\right), f_{0}: \Re \rightarrow \Re^{2}$, that maps a single co-contraction level, $u_{c 0} \in[0,1]$, into the activation levels that produce zero net torque is defined.

From this point, the low-frequency stiffness for those co-contraction levels defined by $f_{0}\left(u_{c 0}\right)$ may be found. It may be computed numerically for each equilibrium point by disturbing the joint angle with respect to the steadystate angle. From $k_{m t f}$ and $k_{m t e}$, one may compute the overall contribution of co-contraction induced by FES to the wrist joint, $k_{c}(\mathbf{c})=k_{m t f}+k_{m t e}$. Figure 7 illustrates the values of $k_{c}$ computed. It can be noted that the curve presents a concave nonlinearity which was not observed in Fig. 6. This is due mainly to nonlinearities in the muscles arms functions provided in [9].

The combination of $f_{0}$, which relates the co-activation levels that produce zero net torque with a scalar control

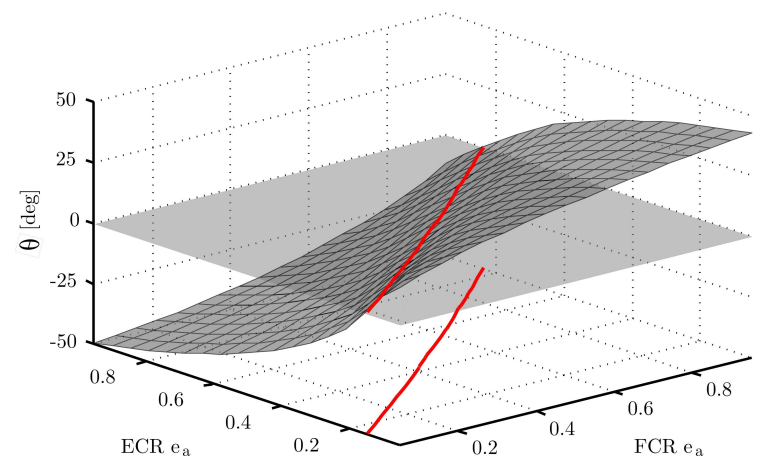

Fig. 6. Equilibrium wrist angles as a function of co-activation levels. A positive $\theta$ refers to flexion of the wrist. The red line indicates the co-activation levels in which the final angle is zero. It has been projected onto the XY plane to facilitate visualization. 


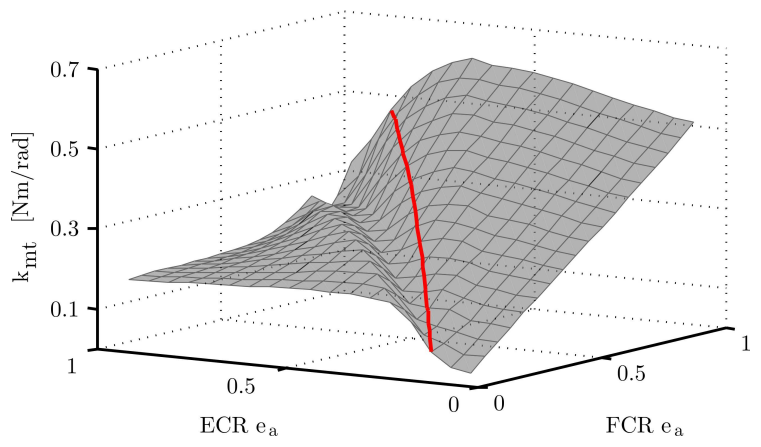

Fig. 7. Muscles contributions to joint stiffness for different coactivation levels. The red line indicates the co-activation levels in which the final angle is zero.

variable, and $k_{c}$, which provides the total contribution of FES-induced co-contraction to joint low-frequency stiffness, may be directly applied into an impedance control scheme. In the context of tremor compensation, in which motion control is not performed, the system is reduced from the complex nonlinear model described in Section II to this simplified description of joint dynamics:

$$
J \ddot{\theta}=-M_{p}-M_{c}+M_{g}+M_{e},
$$

where $M_{c}$, related to co-contraction, is given by

$$
M_{c}=k_{c}\left(f_{0}\left(u_{c 0}\right)\right) \theta
$$

This representation is similar to related works that attempt to model co-contraction contribution to joint stiffness. However, in [12], for instance, the stiffness increases linearly with muscle excitation, while here the stiffness modulation is described by the nonlinear function $f_{0}(\mathbf{c})$, which takes into account different nonlinearities present in the model described in Section II.

\section{CONClusions AND Future WORKS}

Effective therapies for pathological tremor do not exist yet. Based of the fact that co-contraction of antagonist muscles is used for tasks that require increased joint stiffness, this paper has explored the possibility of tremor compensation via FES-controlled co-contraction of the muscles that act on the trembling joint.

In order to fully apprehend the muscles contribution to joint impedance, a detailed nonlinear model of the wrist joint has been developed. The model takes into account different properties that affect muscle force dynamics, such as proprioceptive feedback and combined muscle activation by FES and natural excitation. In addition, it may be also used to simulate different tremor compensation strategies, including strategies based on motion (such as [3]) and impedance control.
Based on the developed model, simulations were performed in order to illustrate tremor reduction via cocontraction of antagonist muscles induced by FES. Furthermore, an analysis of co-contraction nonlinear contribution to joint stiffness was conducted. This result is important for the design of an automatic tremor compensation device that controls stimulation parameters based on motion measurements from a time-varying tremor.

Ongoing effort is focused on the analysis of cocontraction contribution to joint damping and the development of a simplified experimental procedure to identify the subject-specific parameters that mostly affect joint impedance modulation by FES-controlled co-contraction.

\section{ACKNowledgments}

The authors gratefully acknowledge the contribution of Charles Fattal, Christian Geny, and Christine Azevedo for the experimental data acquisition.

\section{REFERENCES}

[1] R. J. Elble and W. C. Koller, Tremor. The John Hopkins University Press, 1990.

[2] E. Rocon, J. M. Belda-Lois, A. F. Ruiz, M. Manto, J. C. Moreno, and J. L. Pons, "Design and validation of a rehabilitation robotic exoskeleton for tremor assessment and suppression," IEEE Transactions on Neural Systems and Rehabilitation Engineering, vol. 15, no. 3, pp. 367-378, 2007.

[3] A. Prochazka, J. Elek, and M. Javidan, "Attenuation of pathological tremors by functional electrical stimulation I: Method," Annals of Biomedical Engineering, vol. 20, no. 2, pp. 205-224, 1992.

[4] D. Zhang, W. T. Ang, and P. Poignet, "Neuromusculoskeletal model exploring peripheral mechanism of tremor," in 30th Annual International Conference of the IEEE Engineering in Medicine and Biology Society, 2008, pp. 3715 - 3719.

[5] C. L. Lynch and M. R. Popovic, "Functional electrical stimulation," IEEE Control Systems Magazine, vol. 28, no. 2, pp. 40-50, Apr. 2008.

[6] M. Ferrarin, F. Palazzo, R. Riener, and J. Quintern, "Modelbased control of FES-induced single joint movements," IEEE Transactions on Neural Systems and Rehabilitation Engineering, vol. 9, no. 3, pp. 245-257, Sep. 2001.

[7] J. M. Winters, "An improved muscle-reflex actuator for use in large-scale neuro-musculoskeletal models," Annals of Biomedical Engineering, vol. 23, no. 4, pp. 359-374, 1995.

[8] G. J. Loren and R. L. Lieber, "Tendon biomechanical properties enhance human wrist muscle specialization," Journal of Biomechanics, vol. 28, no. 7, pp. 799-799, 1995.

[9] M. A. Lemay and P. E. Crago, "A dynamic model for simulating movements of the elbow, forearm, and wrist," Journal of Biomechanics, vol. 29, no. 10, pp. 1319-1330, 1996.

[10] N. Wenderoth and O. Bock, "Load dependence of simulated central tremor," Biological Cybernetics, vol. 80, no. 4, pp. 285290, 1999.

[11] S. Mohammed, D. Guiraud, and P. Poignet, "Closed loop nonlinear model predictive control applied on paralyzed muscles to restore lower limbs functions," in IEEE/RSJ International Conference on Intelligent Robots and Systems, 2006, pp. 259 -264 .

[12] N. Hogan, "Adaptive control of mechanical impedance by coactivation of antagonist muscles," IEEE Transactions on Automatic Control, vol. 29, no. 8, pp. 681-690, Aug. 1984.

[13] H. Park and D. M. Durand, "Motion control of musculoskeletal systems with redundancy," Biological Cybernetics, vol. 99, no. 6 , pp. 503-516, 2008. 